\title{
hsa-miR-149-5p Diminish MAPK and PI3K/Akt Signalling Pathways through Down-regulation of ERbB3 in SLE Patients
}

Ghods FJ ${ }^{1 *}$, Sarikaya $A T^{2}$, Arda $\mathbf{N}^{1}$ and Hamuryudan $\mathrm{V}^{3}$

${ }^{1}$ Department of Molecular Biology and Genetics, Faculty of Science, Istanbul University, Istanbul, Turkey

${ }^{2}$ Department of Molecular Biology and Genetics, Faculty of Arts and Sciences, Yeni Yuzyil University, Istanbul, Turkey

${ }^{3}$ Department of Rheumatology, Cerrahpasa Faculty of Medicine, Istanbul University, Istanbul, Turkey

\begin{abstract}
Introduction: In Systemic Lupus, miRNAs construct a substantial layer of post-transcriptional gene expression regulation. Availability of sensitive and specific methods for their detection makes them candidates for potential biomarker discovery. Here we compared miRNAs particular to SLE patients with healthy controls.
\end{abstract}

Methods: Total RNA and miRNAs were isolated from blood and serum of 16 SLE patients and 8 healthy controls for microarray assays. Potential target genes were predicted and interrogated with mRNA profiling data. BLAST alignment analysis was done between differentially expressed miRNAs and predicted target genes. Microarray results were confirmed by QRT-PCR.

Results: 10 miRNAs were differently expressed in SLE patients of which, has-miR-149-5p was up-regulated about 8.5 fold. Among predicted targets only ERBB3 approved by mRNA profiling and found to be down-regulated approximately by two fold. BLAST alignment analysis of mature sequence of has-miR-149-5p and ERbB3 sequence revealed that 16 of 18 nucleotides belong to $h s a-m i R-149-5 p$, matched with nucleotides in minus chain of target gene (89\%). Two nucleotides "mismatches" did not interfere with target mRNA degradation.

Conclusion: We conclude $h s a-m i R-149-5 p$ degrades ERbB3 gene's primary transcript before splicing meaning that up-regulation of $h s a-m i R-149-5 p$ activates direct/indirect apoptosis by stopping $E R b B 3$ translation and could be a biomarker candidate for lupus activity.

Keywords: hsa-miR-149-5p; ERbB3; Systemic Lupus Erythematosus; biomarker

\section{Introduction}

MiRNAs are small, approximately 20 nucleotides in length, nonprotein-coding single strand RNAs. Their ability to regulate gene expression post-transcriptionally by binding to target mRNAs with low specificity leading to inhibition of gene expression through targeted mRNA degradation, mRNA cleavage, or translational arrest is the hallmark of miRNAs [1-3]. So miRNAs manifest temporal tissuespecific gene expression patterns [4]. MiRNAs are transcribed by RNA polymerase II and related transcription factors. After passing through a lot of control layers, eventually biogenesis cycle of miRNAs has been completed and they are converted to functional mature molecules [5].

Systemic Lupus Erythematosus (SLE) represents the prototype of human autoimmune diseases. The etiology of this autoimmunity disorder remains unknown but autoantibody production and immune complex formation that lead to intense inflammation and multiple organ damage were determined in it [6]. SLE has a prevalence of $\sim 40$ cases per 100,000 individuals with onset typically occurring in women of childbearing age. Increasing evidence has shown that aberrant expression of miRNAs is involved in the pathogenesis of the lupus [7-10].

According to the significant role of miRNAs in regulation of signaling pathways and functions of the immune system elements, such as cytokines, in this study we aimed to investigate one or a group of miRNAs particular to SLE patients compared with healthy control group. This approach will provide novel insights into the effects of miRNAs on disease activity and contribute to development of biomarkers for the diagnosis and treatment assessment.

\section{Clinical Significance}

Growing evidence demonstrated that miRNAs can function as regulatory elements on signaling pathways and play significant roles in immune responsing cells. Serum miRNAs would be as early promise as non-invasive biomarkers for disease activity, different organs involvement and treatment monitoring assessments in complex autoimmune diseases such as Systemic Lupus Erythematosus (SLE).

\section{Material and Methods}

\section{Study participants and sample processing}

In this study a total of 24 subjects, including 8 healthy volunteers, 16 SLE patients were participated. The mean age for healthy control group was $38.1 \pm 10.5$ and for SLE patients was $39.5 \pm 12.05$. After obtaining the Ethics approval from the Clinical Research Ethics Committee of the Istanbul Faculty of Medicine and written informed consents, $5 \mathrm{~mL}$ blood was collected from each participant. After twice centrifugation at $1900 \mathrm{x} \mathrm{g}$ for $10 \mathrm{~min}$ at $4^{\circ} \mathrm{C}$ and at $16000 \mathrm{xg}$ for $10 \mathrm{~min}$ at $4^{\circ} \mathrm{C}$, their serum supernatant were collected and aliquoted in $1.5 \mathrm{~mL}$ tubes then stored at $-20^{\circ} \mathrm{C}$. Peripheral Blood Mononuclear Cells (PBMC) were isolated according to Ficoll-Hypaque density gradient method $[11,12]$.

\section{miRNA and total RNA extraction}

Total RNA isolated from PBMCs using "RNeasy Mini Kit” (Qiagen) and miRNA isolation from serum samples was done using "mirVana PARIS” (Ambion), according to the manufacturer's instruction. Both

*Corresponding author: Ghods FJ, Department of Molecular Biology and Genetics, Faculty of Science, Istanbul University, Turkey, Tel: +90 (212) 45557 00; E-mail: farghods@gmail.com.

Received November 01, 2018; Accepted November 18, 2018; Published November 30, 2018

Citation: Ghods FJ, Sarikaya AT, Arda N, Hamuryudan V (2019) hsa-miR-149-5p Diminish MAPK and PI3K/Akt Signalling Pathways through Down-regulation of ERbB3 in SLE Patients. Mol Biol 8: 224. doi: 10.4172/2168-9547.1000224

Copyright: $\odot 2019$ Ghods FJ, et al. This is an open-access article distributed under the terms of the Creative Commons Attribution License, which permits unrestricted use, distribution, and reproduction in any medium, provided the original author and source are credited. 
miRNA and total RNA samples analyzed by bioanalyzer using "RNA 6000 Nano kit" and "Small RNA Kit" (Agilent) respectively, according to the manufacturer's instructions. In the case of total RNA, RIN numbers equal to or higher than 7.5-8 were acceptable. Aliquots of the both miRNA and total RNA samples were used for all the experiments in both the microarray and RT-qPCR measurements.

\section{Microarray analysis}

Samples were labelled with $\mathrm{Cy} 3$ and then heat denatured and hybridized to Agilent 8 x $60 \mathrm{~K}$ miRNA microarrays V19 comprised of 2006 probes targeting a comprehensive selection of human miRNAs alongside with virus miRNAs and control probes from Sanger miRBase at $37^{\circ} \mathrm{C}$ for $20 \mathrm{~h}$. After hybridization and washing processes, slides were scanned in Agilent Microarray Scanner (Agilent Technologies). For further analysis Gene Spring software was used and the common deregulated miRNAs presented in serum samples were listed in Table 1.

For mRNA microarray assay Agilent Sure Print G3 Human Gene Expression Microarray v2 (G4851B, Agilent Technologies) was used in which $200 \mathrm{mg}$ of total RNA per each sample was labelled and hybridized according to the manufacturer's instructions. Image acquisition and feature extraction were as described for miRNA microarrays.

\section{Target Prediction}

In order to reject the potential false positive rates resulted from the single use of the each software, only the targets that were approved and predicted by microRNAorg, TargetScan and PITA prediction tools at the same time, were considered [13-16]. Only cytokine related genes and related pathways were taken into the account since the main goal of the study was to examine the effects of cytokines on the etiology of the SLE. The KEGG pathway database package presented 229 pathways were used in this study $[17,18]$.

\section{BLAST Alignment Analysis between Differentially Expressed miRNAs and Predicted Potential Target Genes'Sequences}

Each candidate miRNA identified by microarray assay used as a separate query (mature miRNA sequences were used). The BLAST (Basic Local Alignment Search Tool) [http://www.ncbi.nlm.nih. gov/BLAST] program was used to find matches in predicted target genes' transcripts sequences at the National Center for Biotechnology Information (NCBI). The outputs from this database search were compared and the best matches from searches were selected based on the length of the match, percentage of identity of match, lack of gaps or deletions and inclusion of the seed sequence.

\section{Validation of mature candidate miRNAs and ERBB3 isoforms by qRT-PCR}

In order to verify the miRNA microarray results, the mature miRNAs were assayed by qRT-PCR. RT-primers purchased from TAG Copenhagen (Denmark) and designed as described for miR-specific RTqPCR primers [19-24]. Following cDNA synthesis, QRT-PCR primers designing was done using "miRprimer" software [24]. Amplification was done using FastStart SYBR Green Master Kit (Roche, Germany) according to the manufacturer's protocol. Reactions were accomplished for each sample at $95^{\circ} \mathrm{C} / 10 \mathrm{~min}$, followed by 40 cycles of $95^{\circ} \mathrm{C} / 15$ sec and $60^{\circ} \mathrm{C} / 1 \mathrm{~min}$ in a Stratagene Mx3000P (MX3000P, Stratagene, USA). The relative amount of each miRNA to reference gene (U6 RNA) measured by the equation $2^{\text {-(CtmiRNA-CtU6) }}$.

In the case of $E R B B 3$ isoforms (s and 1), the total RNA was reverse transcribed to CDNA and transcript levels of them were measured in a Stratagene Mx3000P (MX3000P, Stratagene, USA). The QRT-PCR primer pairs designed using Primer-BLAST [25] are listed in Table 2. Amplification was done using FastStart SYBR Green Master Kit (Roche, Germany) according to the manufacturer's instruction. The real-time PCR reactions were performed for each sample at $95^{\circ} \mathrm{C} / 5 \mathrm{~min}$ as an initial polymerase activation step; and then 45 amplification cycles at $94^{\circ} \mathrm{C} / 15 \mathrm{sec}$ and $59^{\circ} \mathrm{C} / 30 \mathrm{sec}$. The relative expression of mRNAs was calculated by $\Delta \Delta \mathrm{CT}$ method.

Statistical tests such as Student $t$ test (2 groups) or ANOVA were used for group-wise comparisons and all of them were performed 2-sided. All statistical analyses was performed using "Statistical Package for the Social Sciences v20.0" (SPSS Inc, Chicago, IL, USA). The significance level of $(\mathrm{p}<0.05)$ was mentioned.

\section{Results}

\section{Identification of SLE-Specific miRNAs in microarray analysis}

Comparing SLE patients group $(\mathrm{N}=16)$ with control group $(\mathrm{N}=8)$, a total of 10 miRNAs were detected by miRNA microarray among which, has-miR-149-5p was up-regulated miRNA compared with control, representing approximately an 8.5 -fold changes. These 10 deregulated miRNAs are demonstrated in Table 3.

\section{Prediction of potential target genes for hsa-miR 149-5p}

Based on prediction by microRNAorg, TargetScan and PITA prediction softwares, $h s a-m i R$ 149-5p was expected to target 142 genes in total.

To explore the role that aberrantly expressed miRNAs play in the

\begin{tabular}{|c|c|c|}
\hline Gene_name & $\begin{array}{c}\text { Forward primer } \\
5^{\prime} 3^{\prime}\end{array}$ & $\begin{array}{c}\text { Reverse primer } \\
5^{\prime} 3^{\prime}\end{array}$ \\
\hline U6* & GCTTCGGCAGCACATATACTAAAAT & CGCTTCACGAATTTGCGTGTCAT \\
\hline hsa-miR-149-5p & GCAGGGGAGTGAAGACAC & GGTCCAGTTTTTTTTTTTTTTTTTCTC \\
\hline hsa-miR-933 & GCAGGGGAGAGGTCTC & GTCCAGTTTTTTTTTTTTTTTAGGGA \\
\hline
\end{tabular}

*indicates reference gene

Table 1: QRT-PCR primer sequences for assayed miRNAs.

\begin{tabular}{|c|c|c|c|}
\hline Gene_name & $\begin{array}{c}\text { Forward primer } \\
5^{\prime} 3^{\prime}\end{array}$ & $\begin{array}{c}\text { Reverse primer } \\
5^{\prime} 3^{\prime}\end{array}$ & Anneal Temp $\left({ }^{\circ} \mathrm{C}\right)$ \\
\hline$G A P D H^{*}$ & ATGGGGAAGGTGAAGGTCG & GGGGTCATTGATGGCAACAATA & 58 \\
\hline$E R B B 3$ (transcript variant s) & CCTGCAGTGGATTCGAGAAGT & ACCTTTAGACAGCGGCCAAG & 59 \\
\hline$E R B B 3$ (transcript variant 1) & TTGACTGGAGGGACATCGTG & AATGAGAAGCCCCGGTTGTAG & 59 \\
\hline
\end{tabular}

*indicates reference gene

Table 2: QRT-PCR primer sequences for isoforms of ERBB3. 
Citation: Ghods FJ, Sarikaya AT, Arda N, Hamuryudan V (2019) hsa-miR-149-5p Diminish MAPK and PI3K/Akt Signalling Pathways through Downregulation of ERbB3 in SLE Patients. Mol Biol 8: 224. doi: 10.4172/2168-9547.1000224

Page 3 of 6

\begin{tabular}{|c|c|c|c|c|c|c|c|}
\hline Systematic_name & FC & Reg & $P$ value & Active_seq & Chr & Comparison & mirbase accession number \\
\hline hsa-miR-149-5p & 8.62 & up & 0.0257 & GGGAGTGAAGACACGGAG & 2 & SLE vs. C & MIMAT0000450 \\
\hline hsa-miR-1825 & 11.17 & up & 0.0406 & GGAGAGGAGGGCAC & 20 & SLE vs. C & MIMAT0006765 \\
\hline hsa-miR-3675-3p & 9.90 & up & 0.0033 & TTGGGGGAGTTCCTTA & 1 & SLE vs. C & MIMAT0018099 \\
\hline hsa-miR-4313 & 5.91 & up & 0.0019 & GGGTTTGGGGCCA & 15 & SLE vs. C & MIMAT0016865 \\
\hline hsa-miR-574-5p & 16 & up & 0.0062 & ACACACTCACACACACAC & 4 & SLE vs. C & MIMAT0004795 \\
\hline hsa-miR-933 & 5.75 & up & 0.0113 & GGGAGAGGTCTCCCT & 2 & SLE vs. C & MIMAT0004976 \\
\hline hsa-miR-1304-3p & 3.68 & up & 0.0472 & GGGGTTCGAGGCT & 11 & SLE vs. C & MIMAT0022720 \\
\hline hsa-miR-2116-3p & 5.59 & up & 0.0376 & GGGAGTTCTTGGCATG & 15 & SLE vs. C & MIMAT0011161 \\
\hline hsa-miR-3195 & 6.75 & up & 0.0364 & AACCCGGGCCCG & 20 & SLE vs. C & MIMAT0015079 \\
\hline hsa-miR-365a-3p & 8.75 & up & 0.021 & ATAAGGATTTTTAAGGGGCATTA & 16 & SLE vs. C & MIMAT0000710 \\
\hline
\end{tabular}

Table 3: List of ten SLE deregulated miRNAs identified by miRNA microarray analysis. FC: Fold Change; Reg: Regulation; SLE: Systemic Lupus Erythematosus; C: Control.

\begin{tabular}{|l|c|c|c|}
\hline Systematic_name & $\boldsymbol{P}$ value & Potential Molecular Targets & Pathway \\
\hline & 0.00000004 & ERBB3, AKT3, BIM & EGbB-Signaling-Pathway \\
\cline { 2 - 3 } & 0.00000004 & REPS2, FOXO4, SP1 & Cytokines-and-Inflammatory-Response \\
\cline { 2 - 3 } hsa-miR-149-5p & 0.00355071 & IL-13 & JAK-STAT, IL-17-Signaling-Pathway \\
\cline { 2 - 3 } & 0.00355071 & SP1 & TAB3 \\
\cline { 2 - 3 } & 0.00355071 & AKT3 & Toll-like-Receptor-Signaling-Pathway \\
\cline { 2 - 3 } & 0.00355071 & Torc2 & AMPK-Signaling-Pathway \\
\cline { 2 - 3 } & 0.00355071 & NFATC3 & B-Cell-Receptor-Signaling-Pathway \\
\hline
\end{tabular}

Table 4: The potential gene targets for the differentially expressed hsa-miR $149-5 p$ in SLE patients compared to healthy controls. $\mathrm{P}$ value $\leq 0.05$ indicates the difference is significant.

\begin{tabular}{|l|c|c|c|}
\hline Name & Fold Change & Fold Change $\boldsymbol{P}$ value & Pathway \\
\hline \multirow{2}{*}{ ERBB3 } & 2.05 & $\leq 0.05$ & ErbB-Signaling-Pathway \\
\cline { 2 - 4 } & 2.07 & $\boldsymbol{P}$ value & \multirow{2}{*}{0.00361} \\
\hline
\end{tabular}

Table 5: Validation of the targets which were predicted for miRNAs with differential expression pattern in SLE patients compare to healthy controls. $\mathrm{P}$ vale $\leq 0.05$ indicates the difference is significant.

cytokine related pathways, we only assigned putative targets of these miRNAs, into wikipathways. Table 4 displays these target genes, and the pathways they are involved. The $p$-value was adjusted as $\leq 0.05$.

\section{Validation of Bioinformatics Prediction Using a Simultaneous mRNA/miRNA Expression Profiling Approach}

To increase the accuracy of bioinformatics' predictions, mRNA expression profiling was done simultaneously. Among the targets which were predicted for hsa-miR 149-5p with differential expression pattern in SLE patients compared to healthy controls only one (6.6 \%) ERBB3 (two polymorphic forms) were found to be down-regulated approximately by two fold as shown in Table 5 .

This finding is logic since the expression level of miRNAs and mRNAs should be inversely correlated based on the fact that miRNAs regulate gene expression by inhibiting translation or inducing deadenylation of mRNAs followed by their degradation.

\section{Analysis of $E R b B 3$ gene and has-miR-149-5p alignment}

To further investigate the role of $h s a-m i R-149-5 p$ in ERbB3 gene regulation, similarity matches were screened by comparing the nucleotide sequence of $h s a-m i R-149-5 p$ with the $E R b B 3$ gene sequence in the "Homo sapiens" genome registered in the NCBI database. The randomness of the regions showing homology according to the obtained alignment was statistically evaluated by BLAST. The expected value (e), which is exponentially related to the similarity value (S) according to the "Gumble Extreme Value Distribution" (EVD) formula, was calculated from the maximum likelihood of nucleotide probabilities. Bit scores are determined by taking logarithms of $S$ value so that errors can be minimized [26]. Since $E$ values less than 0.1 or 0.05 are typically taken to represent biological significance and indicate that the alignment has not occurred by chance, $E$ values smaller than 0.05 were selected from the output of the BLAST search [27]. The similarity of sequences over 50 is considered significant for the bit score $[27,28]$. So, among the results the one in which the $E$ score was (0.003), with the bit score of (26.3 ' 13 ') and with the highest "Query coverage" value (72.2\%) was selected.

Base pairing region of has-miR-149-5p with its target mRNA, ErbB3, was located between nucleotides at position 9144-9156 on mRNA and was belonged to the intronic region as shown in Figure 1.

\footnotetext{
9001 cagggtcaggggcaggtggtgttctgtggatagtgcaaggtcagcagggactagtgcaga

9061 gagaaacctgaggaccaagaggttacctggggagatgaggaaggggccctactggtatga

9121 ggcactttgaggagaaagctgcctgtcttcactcccagaagtgacacagcagtgtgacac

9181 agtctactccctactcccaaataggaattagcaagagttaaggccaggtg cagtggetca
}

Figure 1: Matching analysis of $h s a-m i R-149-5 p$ with the sequence between 9144 and 9156 positions of the ErbB3 gene in the genome of "Homo sapiens" registered in the NCBI database. 


\section{hsa-miR-149-5p $\quad 1$ Sequence \\ 13

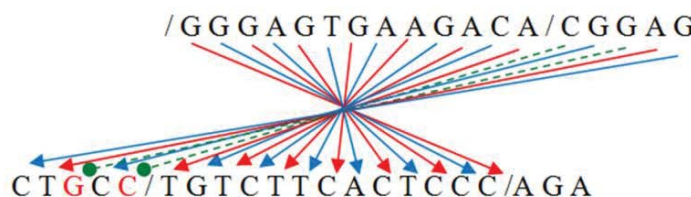 \\ 9161 \\ 9156 \\ 9144 \\ $E R b B 3$ gene}

Figure 2: The hsa-miR-149-5p and ErbB3 gene sequence matching. 13 nucleotides out of the 18 nucleotides belonging to $h s a-m i R-149-5 p$ bind to the target gene's minus (-) chain by $100 \%$. Among 18 nucleotides, only at positions 9157 and $9159(2 \mathrm{nt})$ matching will not occur.

Findings from this study are of great importance in that the miRNA encoded by $h s a-m i R-149-5 p$ breaks down the target mRNA prior to the clipping phase. As shown in Figure 2, 16 of the 18 nucleotides belonging to $h s a-m i R-149-5 p$ match with the nucleotides in the minus (-) chain of the target gene (89\%). It has also been determined that $\mathrm{C}$ nucleotide at position 9157 and nucleotide G at position 9159 "mismatches" do not interfere with target mRNA degradation.

\section{Validation step by quantitative real-time PCR}

However a few differences in the fold changes detected between the miRNA microarray and qRT-PCR results, qPCR results confirmed a similar expression pattern of selected miRNAs. when SLE patients were compared with healthy control group, the fold changes of hsamiR-149-5p and $h s a-m i R-933$ in microarray test were +8.62 and +5.75 (up-regulation) respectively, while in qRT-PCR test they were +7.15 ( $P$ value $=0.004)$ and $+4.36(P$ value $=0.007)$. Also, $\mathrm{qRT}-\mathrm{PCR}$ validated a similar expression patterns for both isoforms of ERBB3 transcript $(P$ value <0.05).

\section{Discussion}

As mentioned above, hsa-miR-149-5p was up-regulated by approximately 8.5 fold changes. Our study did not reproduce microarray analysis results reported by Te et al. In which $h s a-m i R-149^{*}$ was found to be down-regulated in EBV transformed cells from African-American SLE patients with nephritis but was not changed in PBMCs of same individuals. Interestingly enough, in the same study, up-regulation of hsa-miR-149* in either SLE European-American individuals and in SLE-affected monozygotic twins samples derived cell lines was reported [29].

Among the predicted target genes obtained from the bioinformatics studies for hsa-miR-149-5p, ERbB3 gene expression level was also confirmed experimentally by mRNA microarray analysis and validated by QRT-PCR assay.

About $90 \%$ similarities, according to NCBI sequence match analysis results, indicate that $h s a-m i R-149-5 p$ regulates $E R b B 3$ gene's primary transcript by degrading it before splicing. In this study it is revealed that the translation of mRNA isoforms ( $\mathrm{s}$ and 1 ) produced by the primary transcript of $E R b B 3$ gene [30] is inhibited by degradation through $h s a-$ miR-149-5p.

In this study one of the target mRNA candidates for hsa-miR149-5p belongs to the ERbB3 gene. ErbB3, Epidermal Growth Factor Receptor (EGFR), is a member of the tyrosine kinases family. ErbB3 is different from other family members due to lack of real kinase activity
[31]. Thus, ErbB3 is only active as a heterodimer complex and shows the highest affinity for ErbB2; the most efficient complex for the activation of the downstream pathways is the ErbB2/ErbB3 heterodimer [32]. The downstream of the ErbB2/ErbB3 heterodimer, PI3K/Akt pathway is induced and plays an important role in the proliferation, survival and invasion of cancer cells $[33,34]$. ErbB2/ErbB3 heterodimer have been shown to play a role in the growth of human lung adenocarcinoma cells via PI3K/Akt pathway activation $[35,36]$. The ERbB3 signal activates the PI3K/AKT/FRAP1/RPS6 pathway leading to Caspase 3 (CASP3) mediated apoptosis and thus contributes to tumor growth [37]. In other words, it allows cancer cells to immortalize by suppressing apoptosis. Many studies revealed that dysregulated apoptosis machinery account for predisposing to lupus in SLE mice models [38-40]. In a recent study by Bischoff et al. using a vector containing the 3'UTR of ERbB3 cloned downstream of the luciferase CDNA, it was shown that $m i R-149$ directly targets $E R b B 3$. They showed that down-regulation of $E R b B 3$ receptor alongside with multiple down-stream signalling molecules reduces the growth factor Heregulin (HRG) responses [41]. Based on the above information and findings it can be postulated that up-regulation of $h s a-m i R-149-5 p$ activates direct or indirect apoptosis by stopping the $E R b B 3$ transcripts translation.

On the other hand, in some previous studies it has been shown that due to diminished phosphorylation of $\mathrm{PKC}_{\delta}$, the phosphorylation decreases also in ERK, MEK and RAF of MAPK pathway in T-cells of patients with active-lupus patients $[42,43]$. Similar results were also found in microarray results. According to the fact that ERK-MAPK pathway regulates the DNA methyltransferasel (Dnmt1) level in these cells [44], it was suggested that aberrant activation of Dnmt1 can contribute to overexpression of LFA-1 making T-cells autoreactive $[42,45,46]$. But the mechanism by which $\mathrm{PKC}_{\delta}$ activation is impaired has remained unknown. Based on our findings, we suggest that decreased $E R b B 3$ expression mediated by $h s a-m i R-149-5 p$ may be fundamental to part of these abnormalities through abrogation of $E R b B 3$ (EGFR) mediated tyrosine phosphorylation of $\mathrm{PKC}_{\delta}$. As a result, impaired T-cell ERK-MAPK pathway $\left(\mathrm{PKC}_{\delta}\right.$ - Ras _ Raf _ MEK_ ERK) seems to be responsible for decreasing Dnmt1 level, inducing auto reactivity in T-cells.

These results do not contradict the accuracy of targets which have been predicted for detected miRNAs in this study, because unavailability of these targets in gene expression profiling data can be explained by the emphasis on the fact that miRNAs regulate gene expression at the post-transcriptional level not only by de adenylation and mRNA decay but also through translational repression. So functional experimental studies are required to verify and establish the association between aberrantly expressed miRNAs and SLE.

\section{Conclusion}

In this study for each case (SLE or Healthy) miRNA and mRNA expression profiling were done separately and without creating of miRNA and mRNA pools. This issue has improved our study's strengths and accuracy.

Based on above findings, we postulate that this hsa-miR-149$5 p$ could be attractive as biomarker candidate for disease activity assessment of lupus and $h s a-m i R-149-5 p$ and ERbB2/ERbB3 signalling pathway may be serving as molecular targets or pathways that can be specifically targeted for therapy. Before accepting the $h s a-m i R-149-5 p$ as a molecular target, the information provided in this study should be extended to further studies with larger sample sizes. 
Citation: Ghods FJ, Sarikaya AT, Arda N, Hamuryudan V (2019) hsa-miR-149-5p Diminish MAPK and PI3K/Akt Signalling Pathways through Downregulation of ERbB3 in SLE Patients. Mol Biol 8: 224. doi: 10.4172/2168-9547.1000224

Page 5 of 6

\section{Acknowledgement}

The authors thank the SLE patients who participated in this study, and Prof. Dr. Gulruh Albayrak (Istanbul University); Assist. Prof. Dr. Emre Yuruk (Yeni Yuzyil University); for their helpful suggestions, and Prof. Dr. Ahad Jafari Ghods, MD. FACP, (Iran University of Medical Sciences) for critical reading and comments on the manuscript.

\section{Funding Details}

This Research Project was partially sponsored by The Scientific Research Projects unit

(BAP) of Istanbul University with grant number (26843).

\section{Declaration of Interest Statement}

The authors whose names are listed above certify that they have no conflict of interest.

\section{Data Availability Statement}

The data that support the findings of this study are available from Gene Spring software

v12.6 (Agilent) but restrictions apply to availability of these data, which were used under license for the current study, and so are not publicly available. Data are however available from the authors upon reasonable request and with permission of Agilent.

\section{References}

1. Ambros V (2003) MicroRNA pathways in flies and worms: growth, death, fat, stress, and timing. Cell 113: 673-676.

2. Mortimer SA, Kidwell MA, Doudna JA (2014) Insights into RNA structure and function from genome-wide studies. Nat Rev Genet 15: 469-479.

3. Rosani U, Pallavicini A, Venier P (2016) The miRNA biogenesis in marine bivalves. Peer J 4: e1763.

4. Carrington JC, Ambros V (2003) Role of microRNAs in plant and anima development. Science 301: 336-338.

5. Shomron N, Golan D, Hornstein E (2009) An Evolutionary Perspective of Animal MicroRNAs and Their Targets. J Biomed Biotechnol 2009: 594738.

6. Adams SG Jr, Dammers PM, Saia TL, Brantley PJ, Gaydos GR (1994) Stress, depression, and anxiety predict average symptom severity and daily symptom fluctuation in systemic lupus erythematosus. J Behav Med 17: 459-477.

7. Zhou X, Jeker LT, Fife BT, Zhu S, Anderson MS, et al. (2008) Selective miRNA disruption in T reg cells leads to uncontrolled autoimmunity. J Exp Med 205 1983-1991.

8. Pauley KM, Cha S, Chan EKL (2009) MicroRNA in autoimmunity and autoimmune diseases. J Autoimmun 32: 189-94.

9. Sonkoly E, Pivarcsi A (2009) Advances in microRNAs: implications for immunity and inflammatory diseases. J Cell Mol Med 13: 24-38.

10. Zigang Qu, Wenhui Li, Baoquan Fu (2014) MicroRNAs in Autoimmune Diseases. BioMed Research International 2014: 527895.

11. Böyum A (1968) Isolation of mononuclear cells and granulocytes from human blood. Scand J Clin Lab Invest Suppl 97: 77-89.

12. Rodrıguez-Gonzalez FG, Mustafa DA, Mostert B, Sieuwerts AM (2013) The challenge of gene expression profiling in heterogeneous clinical samples. Methods 59: 47-58.

13. Huang JC, Babak T, Corson TW, Chua G, Khan S, et al. (2007) Using expression profiling data to identify human microRNA targets. Nat Methods 4: 1045-1049.

14. Huang $Y$, Zou $Q$, Song $H$, Song F, Wang L, et al. (2010) A study of miRNAs targets prediction and experimental validation. Protein Cell 1: 979-986.

15. Alexiou P, Maragkakis M, Papadopoulos GL, Reczko M, Hatzigeorgiou AG (2009) Lost in translation: an assessment and perspective for computational microRNA target identification. Bioinformatics 25: 3049-3055.

16. Liu X, Wang J, Sun G (2015) Identification of key genes and pathways in renal cell carcinoma through expression profiling data. Kidney Blood Press Res 40 288-297.

17. Carlson M (2016) A set of annotation maps for KEGG. KEGG db.

18. Kanehisa M, Goto S, Sato Y, Kawashima M, Furumichi M (2014) Data, information, knowledge and principle: Back to metabolism in KEGG. Nucleic Acids Res 42: D199-D205

19. Ding SW, Voinnet O (2007) Antiviral immunity directed by small RNAs. Cell $130: 413-426$.

20. Lecellier CH, Dunoyer P, Arar K, Lehmann-Che J, Eyquem S (2005) A cellular microRNA mediates antiviral defense in human cells. Science $308: 557-560$

21. Umbach JL, Cullen BR (2009) The role of RNAi and microRNAs in animal virus replication and antiviral immunity. Genes Dev $23: 1151-1164$

22. Balcells I, Cirera S, Busk PK (2011) Specific and sensitive quantitative RT-PCR of miRNAs with DNA primers. BMC Biotechnol 11.

23. Busk PK (2010) Method for Quantification of Small RNA Species. WO/2010/ 085966.

24. Busk PK (2014) A tool for design of primers for microRNA-specific quantitative RT-qPCR. BMC Bioinformatics 15.

25. Ye J, Coulouris G, Zaretskaya I, Cutcutache I, Rozen S, et al. (2012) PrimerBLAST: A tool to design target-specific primers for polymerase chain reaction. BMC Bioinformatics $13: 134$.

26. Altschul SF, Madden TL, Schaffer AA, Zhang J, Zhang Z, et al. (1997) Gapped BLAST and PSI-BLAST: A new generation of protein database search programs. Nucleic Acids Res 25: 3389-3402.

27. Pertsemlidis A, Fondon III JW (2001) Having a BLAST with bioinformatics (and avoiding BLASTphemy). Genome Biol 2.

28. Villareal D, Nirde P, Hide M, Barnabe C, Tibayrenc M, et al. (2005) Differentia gene expression in benznidazole-resistanat Trypanosoma cruzi parasites. Antimicrob Agents Chemother 49: 2701-2709.

29. Te JL, Dozmorov IM, Guthridge JM, Nquyen KL, Cavett JW, et al. (2010) Identification of unique microRNA signature associated with lupus nephritis. PLoS One 5:e10344.

30. Katoh M, Yazaki Y, Suqimura T, Terada M (1993) c-erbB3 gene encodes secreted as well as transmembrane receptor tyrosine kinase. Biochem Biophys Res Commun 192: 1189-1197.

31. Guy PM, Platko JV, Cantley LC, Cerione RA, Carraway KL (1994) Insect cellexpressed $\mathrm{p} 180$ erbB3 possesses an impaired tyrosine kinase activity, USA. Proc Natl Acad Sci 91: 8132-8136.

32. Yarden $Y$, Sliwkowski MX (2001) Untangling the ErbB signalling network. Nat Rev Mol Cell Biol 2: 127-137.

33. Arboleda MJ, Lyons JF, Kabbinavar FF, Bray MR, Snow BE, et al. (2003) Overexpression of AKT2/protein kinase Bbeta leads to up-regulation of beta1 integrins, increased invasion, and metastasis of human breast and ovarian cancer cells. Cancer Res 63: 196-206.

34. Holbro T, Beerli RR, Maurer F, Koziczak M, Barbas CF, et al. (2003) The ErbB2/ ErbB3 heterodimer functions as an oncogenic unit: ErbB2 requires ErbB3 to drive breast tumor cell proliferation. Proc Natl Acad Sci USA 100: 8933-8938.

35. Sithanandam G, Smith GT, Masuda A, Takahashi T, Anderson LM, et al. (2003) Cell cycle activation in lung adenocarcinoma cells by the ErbB3/ phosphatidylinositol 3-kinase/Akt pathway. Carcinogenesis 24: 1581-1592.

36. Sithanandam G, Fornwald LW, Fields J, Anderson LM (2005) Inactivation of ErbB3 by siRNA promotes apoptosis and attenuates growth and invasiveness of human lung adenocarcinoma cell line A549. Oncogene 24: 1847-1859.

37. Lee D, Yu M, Lee E, Kim H, Yang Y, et al. (2009) Tumor-specific apoptosis caused by deletion of the ERBB3 pseudo-kinase in mouse intestinal epithelium. J Clin Invest 119: 2702-2713.

38. Nagata S, Suda T (1995) Fas and Fas ligand: Ipr and gld mutations. Immuno Today 16: 39-43

39. Napirei M, Karsunky H, Zevnik B, Stephan H, Mannherz HG, et al. (2000) Features of systemic lupus erythematosus in Dnase1- deficient mice. Nat Genet 25: 177-181.

40. Botto M, Dell'Agnola C, Bygrave AE, Thompson EM, Cook HT, et al. (1998) Homozygous $\mathrm{C} 1 \mathrm{q}$ deficiency causes glomerulonephrities associated with multiple apoptotic bodies. Nat Genet 19: 56-59.

41. Bischoff A, Bayerlová M, Strotbek M, Schmid S, Beissbarth T, et al. (2015) A global microRNA screen identifies regulators of the ErbB receptor signaling network. J Cell Commun Signal 13. 
Citation: Ghods FJ, Sarikaya AT, Arda N, Hamuryudan V (2019) hsa-miR-149-5p Diminish MAPK and PI3K/Akt Signalling Pathways through Downregulation of ERbB3 in SLE Patients. Mol Biol 8: 224. doi: 10.4172/2168-9547.1000224

Page 6 of 6

42. Gorelik G, Richardson B (2008) Aberrant T cell ERK pathway signaling and chromatin structure in lupus. Autoimmun Rev 8: 196-198.

43. Gorelik G, Fang JY, Wu A, Sawalha AH, Richardson B, et al. (2007) Impaired T cell protein kinase $\mathrm{C} \delta$ activation decreases ERK pathway signaling in idiopathic and hydralazine-induced lupus. J Immunol 179: 5553-5563.

44. Deng C, Kaplan MJ, Yang J, Ray D, Zhang Z, et al. (2001) Decreased Rasmitogen-activated protein kinase signaling may cause DNA hypomethylation in T lymphocytes from lupus patients. Arthritis Rheum 44: 397-407.
45. Richardson B, Scheinbart L, Strahler J, Gross L, Hanash S, et al. (1993) Evidence for impaired T cell DNA methylation in systemic lupus erythematosus and rheumatoid arthritis. Arthritis Rheum 33: 1665-1673.

46. Oelke K, Richardson B (2004) Decreased T cell ERK pathway signaling may contribute to the development of lupus through effects on DNA methylation and gene expression. International reviews of immunology 23: 315-331. 